\title{
Intermolecular interactions between thiocyanato ligands in metal complexes
}

\author{
Jorge Echeverría \\ Departament de Química Inorgànica i Orgànica and Institut de Química Teòrica i \\ Computacional IQTC-UB, Universitat de Barcelona, Martí i Franquès 1-11, 08028 \\ Barcelona (Spain). \\ e-mail: jorge.echeverria@qi.ub.es
}

\section{SUPPORTING INFORMATION}


Table S1. CSD refcodes lists found for any of the interaction typologies studied in this article.

\begin{tabular}{|c|c|c|c|c|c|}
\hline $\mathrm{N}-\mathrm{C}$ & S-C & S-N & N-CS & S-CS & S-CN \\
\hline ACUGEN01 & ACUGEN & ACOXRH & EHUGIY & AXENU001 & BOVLUU \\
\hline AGESAH & AGESAH & BUWXAS & EMENUF & BUGJEU & BUYCUU \\
\hline EXUGIP & AXENUO & BUYDEF & FOTNAF & CURZUL & BUYDAB \\
\hline EXUGOV & DERQIA & ENHGTC10 & GARREY & DERQIA01 & CECZAM \\
\hline FIMJOD & EBEBUK & ILETOI & MAJGAH & MASLIE & CEFCEX \\
\hline GEZSOW & ETARAS & JAVPAX & NAMLIU & MASLOK & ETARAS \\
\hline HOSQEM & EXUGOV & MASLIE & NEYJIK & NEYJIK & IXUKER \\
\hline LERFUL & GABNEC & MASLOK & OCOYIR & RATKAA & KEJCUA \\
\hline MEPHUN & HEDWIW & RATCIA & RATBOF & & KITBOE \\
\hline MOJBIX & HOSQEM & WUYDUR & RATCOG & & MEPHUN \\
\hline OCOYIR & HUYJUH & & & & OKAPEV \\
\hline RATCEW & JAVPAX & & & & PUZNEF \\
\hline REMMEC & KUJWIX & & & & RATCIA \\
\hline SAKNOI & MASLEA & & & & SEZB0010 \\
\hline SEZB0010 & MOZLIW & & & & SILNIK \\
\hline SILNIK & NELBUB & & & & TORMIW \\
\hline TPARHG10 & OHOCOE & & & & TORMIW01 \\
\hline WEMTOX & PIXYOL & & & & UNOCEG \\
\hline \multirow[t]{4}{*}{ WUYFAZ } & RATCEW & & & & UNOCEG01 \\
\hline & REMMEC & & & & UWIGIR \\
\hline & TENHGT & & & & WEMTOX \\
\hline & ZASLOU01 & & & & ZAVLEQ \\
\hline
\end{tabular}




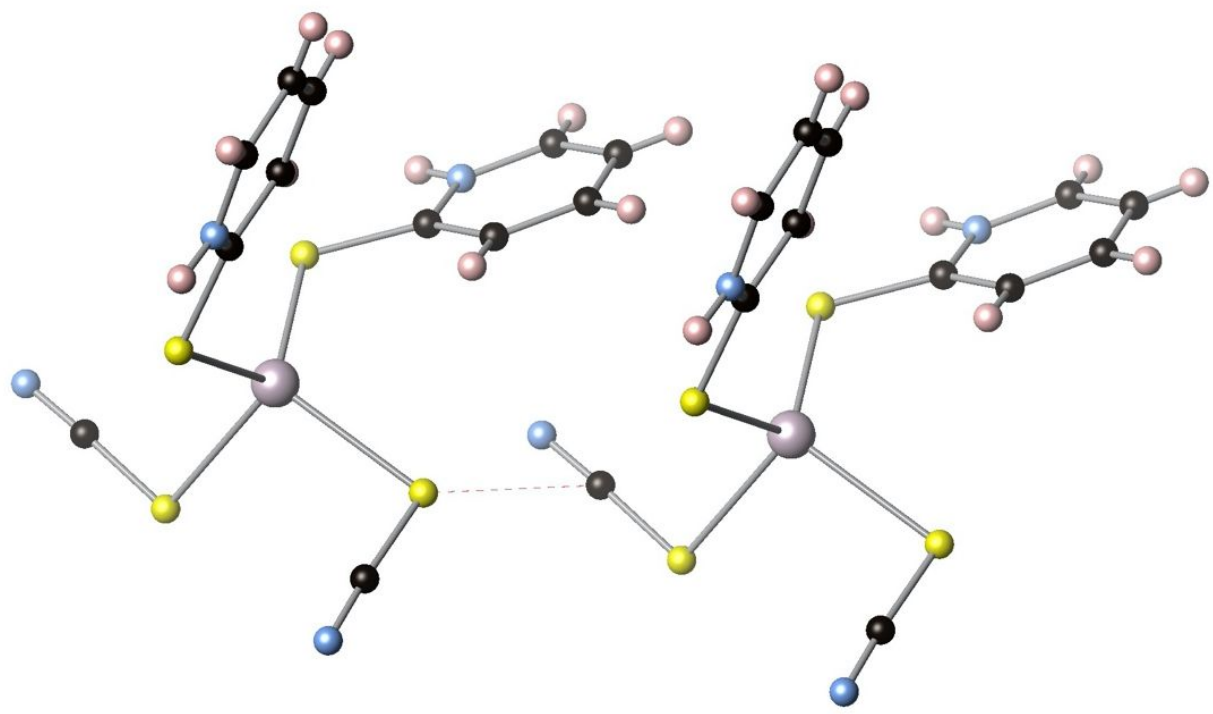

Figure S1. Dimer found in the crystal structure of DERQIA. The intermolecular S...C distance indicated with a dashed line is $3.595 \AA$. 